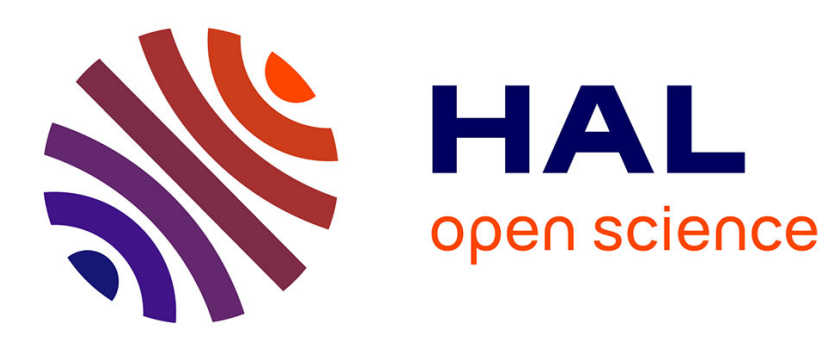

\title{
A Bayesian Framework for Preventive Assistance at Road Intersections
}

\author{
Alexandre Armand, David Filliat, Javier Ibañez-Guzman
}

\section{To cite this version:}

Alexandre Armand, David Filliat, Javier Ibañez-Guzman. A Bayesian Framework for Preventive Assistance at Road Intersections. 2016 IEEE Intelligent Vehicles Symposium (IV'16), Jun 2016, Göteborg, Sweden. hal-01319046

\section{HAL Id: hal-01319046 \\ https://hal.science/hal-01319046}

Submitted on 23 May 2016

HAL is a multi-disciplinary open access archive for the deposit and dissemination of scientific research documents, whether they are published or not. The documents may come from teaching and research institutions in France or abroad, or from public or private research centers.
L'archive ouverte pluridisciplinaire HAL, est destinée au dépôt et à la diffusion de documents scientifiques de niveau recherche, publiés ou non, émanant des établissements d'enseignement et de recherche français ou étrangers, des laboratoires publics ou privés. 


\title{
A Bayesian Framework for Preventive Assistance at Road Intersections
}

\author{
Alexandre Armand ${ }^{1,2}$, David Filliat ${ }^{2}$, Javier Ibañez-Guzman ${ }^{1}$
}

\begin{abstract}
Modern vehicles embed an increasing number of Advanced Driving Assistance Systems (ADAS). Whilst such systems showed their capability to improve comfort and safety, most of them provide assistance only as a last resort, that is, they alert the driver or trigger automatic braking only when collision is imminent. This limitation is mainly due to the difficulty to accurately anticipate risk situations in order to provide the driver with preventive assistance, i.e. assistance allowing for comfortable reaction. This paper presents a Bayesian framework which aims to detect risk situations sufficiently early to trigger conventional curative assistance as well as preventive assistance. By taking into consideration the context, the vehicle state, the driver actuation and the manner how the driver usually negotiates given situations, the framework allows to infer which type of assistance is the most pertinent to be provided to the driver. The principles of this framework are applied to a fundamental case study, the arrival to a stop intersection. Results obtained from data recorded under controlled conditions are presented. They show that the framework allows to coherently detect risk situations and to identify what assistance, including preventive assistance, is the most appropriate for the situation.
\end{abstract}

\section{INTRODUCTION}

Accessibility to motor vehicles has provided freedom of movement to people and changed society. However, they have also brought road accidents which kill more than 1.2 millions people per year in the world. Statistics show that the great majority of accidents are caused by human errors, favored by factors such as distractions, tiredness, etc. [12]. Among all accidents, over $40 \%$ of collisions and $20 \%$ of fatalities happen at road intersections which represent the most complex areas where various entities converge [9].

The problem of road safety has been of much concern for several years in the automotive industry. The first solution to road fatalities was to embed passive safety features in vehicles, such as airbags, ABS, to diminish the consequences of accidents. More recently, encouraged by the extensive progress in robotics and the diminution of sensors costs, intelligent systems progressively took place in vehicles to assist drivers when collisions are imminent. For instance, systems such as Automatic Emergency Braking (AEB) allow to avoid collisions with other vehicles or pedestrians. Whilst such functions allow to avoid collisions, they are triggered as a last resort only.

This paper proposes a Bayesian Network framework that aims to detect risk situations with more anticipation than

\footnotetext{
1 Renault S.A.S, 1 av. du Golf, 78288 Guyancourt, France. alexandre.armand@ renault.com, javier.ibanez-guzman@ renault.com.

2 ENSTA ParisTech/ INRIA FLOWERS team, 828 Boulevard des Maréchaux, 91762 Palaiseau Cedex, France. david.filliat@enstaparistech.fr.
}

conventional systems. The purpose is to infer risk situations early enough to provide preventive assistance (which can be provided in the form of advice) allowing the driver to have sufficient time to react and decelerate comfortably. Further, it allows to identify which type of assistance between AEB, warning alerts and advice is the most pertinent to be triggered, with respect to the situation and the moment at which risk has been detected. The framework is applied for a vehicle that is approaching to a stop intersection.

The reminder of the paper is organized as follows. Section 2 presents a review of literature focusing on risk estimation at road intersections, followed by the problem statement. The Bayesian Network is detailed in Section 3, and Section 4 presents the results from experimentation. Finally, Section 5 concludes the paper.

\section{Related Work ANd Problem Statement}

\section{A. Related Work}

Safety at road intersections is of much concern, and extensive work has been carried out for several years. A review of the related literature allows to identify two major approaches to perform risk assessment: 1) Collision prediction 2) Detection of unexpected manoeuvres.

The first approach aims to perform risk assessment in two steps: trajectory prediction, followed by collision detection. Whilst detection of imminent collisions is mainly performed through the computation of the popular Time To Collision (TTC) [1], there are several methods to predict the trajectory of the subject vehicle. The simplest consists in predicting the vehicle trajectory using conventional motion models [11]. The main drawback of such methods is the difficulty to take into consideration the context and other road users. More sophisticated methods allow to tackle these limits, for instance by using sets of context aware trajectory prototypes, defined by parametric functions [8], or non parametric functions such as Gaussian Processes [6]. The current vehicle trajectory is compared to the set of trajectory prototypes, and the closest prototype is selected to predict the trajectory. Another solution consists of the estimation of the manoeuvre that the vehicle is performing, followed by the estimation of the future vehicle trajectory according to this ongoing manoeuvre. Plenty of techniques exist for manoeuvre estimation, such as Hidden Markov Models (HMM) [4], Support Vector Machine (SVM) [2], neural networks [10], or also Scenario Model Trees (SMT) [5].

The main drawback of the first approach is that trajectories can be accurately predicted over short time horizons 
only, which prevents from accurately predicting collisions several seconds before they occur. The second approach for risk assessment is not based on collision detection, but is higher level as it is based on the detection of unexpected manoeuvres. This context aware approach consists in detecting differences between the manoeuvre that the driver is expected to perform, with the manoeuvre that he is likely to have intention to perform. This approach has been implemented within a Dynamic Bayesian Network and tested in cooperative vehicles [7]. The results of the field trials proved the capability of the model to accurately detect risk situations.

\section{B. Problem Statement}

The literature has shown that multiple techniques allow for the inference of likely collisions in road intersection contexts. However, most of the techniques infer that the situation is likely to be at risk only when the situation has already become dangerous, i.e. when accident is imminent. They can be classified as curative systems as they allow to trigger warning alerts or AEB, leading to driver discomfort. By contrast, preventive systems would aim to assist the driver early enough to allow him to react and avoid the situation to become dangerous. For example, an HMI could help the driver identify the pertinent context entities he is likely to have missed, and guide him to take good decisions. Providing the driver with curative or preventive assistance should depend on the situation, and on the moment at which risk is detected. Actually, triggering AEB too early is not pertinent, as well as triggering advice assistance too late.

In this paper, it is proposed to take benefits from context information, the vehicle state, the driver actuation and the driver individualities to infer risks as the vehicle approaches to a stop intersection. For this purpose, the Bayesian framework that was presented by [7] was extended to detect risk sufficiently early to trigger preventive assistance. Moreover, it allows to identify which of the three main types of assistance is the most pertinent to be provided to the driver, with respect to the situation and the moment at which risk is detected.

\section{BAYESIAN NETWORK}

\section{A. Variables Definition}

Figure 1 shows the proposed Bayesian Network. It stores five conjunction nodes which are presented bellow.

1) Vehicle Physical State: This node stores four variables such as $\phi_{t}^{n}=\left(S_{t}^{n}, P_{t}^{n}, B_{t}^{n}, G_{t}^{n}\right)$. They represent the true physical state of vehicle $n$ at time $t$ :

- $S_{t}^{n} \in \mathbb{R}$, the true speed of vehicle $n$.

- $P_{t}^{n} \in \mathbb{R}$, the true pose of vehicle $n$, which is represented in one dimension as the distance to the coming intersection (i.e. curvilinear abscissa).

- $B_{t}^{n} \in\{$ on, off $\}$, the true state of the brake pedal of vehicle $n$. It is assumed that the state of the brake pedal is binary. State on means that the driver pushes the

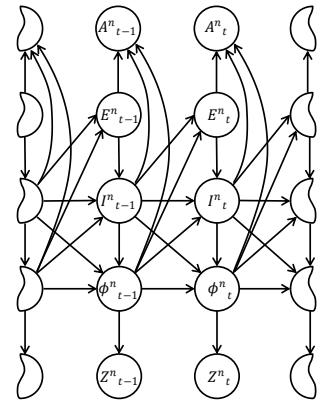

Fig. 1: The proposed Bayesian Network

brake pedal, and state off means that the driver does not push the brake pedal.

- $G_{t}^{n} \in\{o n, o f f\}$, the true state of the gas pedal of vehicle $n$. In the same manner as for the brake pedal, the state of the brake pedal is assumed to be binary.

2) Observations $Z_{t}^{n}$ : This node stores four variables such as $Z_{t}^{n}=\left(\operatorname{Sobs}_{t}^{n}, \operatorname{Pobs}_{t}^{n}\right.$, Bobs $_{t}^{n}$, Gobs $\left._{t}^{n}\right)$. They represent the measurements of the physical state of vehicle $n$ at time $t$ :

- $S^{n}{ }^{0 b s_{t}} \in \mathbb{R}$ the measured vehicle speed of vehicle $n$.

- $P^{n} o b s_{t} \in \mathbb{R}$ the measured pose of vehicle $n$.

- $B^{n} o b s_{t} \in\{$ on, of $f\}$, the observed state of the brake pedal of vehicle $n$.

- $G^{n} o b s_{t} \in\{$ on, off $\}$, the observed state of the gas pedal of vehicle $n$.

3) Intended Manoeuvre $I_{t}^{n}$ : This node stores two variables such as $I_{t}^{n}=\left(I M_{t}^{n}, I R_{t}^{n}\right)$ which represent the manoeuvres that the driver has the intention to perform. The term "manoeuvre" concerns either the vehicle or the driver. A manoeuvre related to the vehicle has to be understood as a the vehicle behaviour impacting its physical state. For example, turning right, or making a stop are considered as manoeuvres related to the vehicle. A manoeuvre related to the driver has to be understood as an action that the driver is performing. For example, reacting by pushing a pedal, or turning the steering wheel are considered as manoeuvres related to the driver.

- $I M_{t}^{n} \in\{$ go, stop $\}$ the intended longitudinal manoeuvre of vehicle $n$. The driver can either have the intention to stop, or to go at the stop intersection.

- $I R_{t}^{n} \in\{$ reaction, no reaction $\}$ the intended manoeuvre of the driver of vehicle $n$. Here, the term "driver manoeuvre" has to be interpreted as the reaction that the driver shows when he starts to interact with the stop intersection. Depending on the context, the driver can therefore have the intention to react, or not to react.

4) Expected Manoeuvre $E_{t}^{n}$ : This node stores two variables such as $E_{t}^{n}=\left(E M_{t}^{n}, E R_{t}^{n}\right)$ which represent the manoeuvres that the driver is expected to perform. These manoeuvres are the same as the intended manoeuvres:

- $E M_{t}^{n} \in\{g o, s t o p\}$ the expected longitudinal manoeuvre of vehicle $n$. The vehicle can either be expected to stop, or expected to go at the stop intersection. 
- $E R_{t}^{n} \in\{$ reaction, no reaction $\}$ the expected manoeuvre of the driver of vehicle $n$. The driver can either be expected to react, or not to react to the stop intersection.

5) Assistance $A_{t}^{n}$ : Assistance can be provided in 3 forms: automatic actuation, warning and advice. Thus, $A_{t}^{n}$, the relevance of providing assistance to the driver of vehicle $n$ at time $t$ is defined by $A_{t}^{n}=\left(A c t_{t}^{n}, \operatorname{War}_{t}^{n}, A d v_{t}^{n}\right)$, with:

- $A c t_{t}^{n} \in\{$ not pertinent,pertinent $\}$ the relevance of performing automatic emergency actuation on vehicle $n$ at time $t$. Act $t_{t}^{n}=$ not pertinent means that automatic actuation at time $t$ is not pertinent and $A c t_{t}^{n}=$ pertinent means that automatic actuation at time $t$ is pertinent.

- $W a r_{t}^{n} \in\{$ not pertinent, pertinent $\}$ the relevance of warning the driver of vehicle $n$ at time $t$. In the same manner as for AEB, warning assistance can either be pertinent or not pertinent.

- $A d v_{t}^{n} \in\{$ not pertinent, pertinent $\}$ the relevance of giving an advice to the driver of vehicle $n$ at time $t$. In the same manner as for AEB, advice assistance can either be pertinent or not pertinent.

\section{B. Parametric Forms}

1) Expected Longitudinal Manoeuvre $E M_{t}^{n}$ : The evolution model of the expected manoeuvre is very simple as the case study consists of the approach to a stop intersection. Thus, the expected longitudinal manoeuvre is always to make a stop at the intersection, independently from the vehicle state and the intended manoeuvre at previous timestep. The conditional probabilities for the expected manoeuvre are therefore defined as follows:

$$
\begin{cases}P\left(E M_{t}=g o\right) & =0 \\ P\left(E M_{t}=\text { stop }\right) & =1\end{cases}
$$

2) Intended Longitudinal Manoeuvre $I M_{t}^{n}$ : The evolution model of the intended longitudinal manoeuvre is the same as the one used in [7]. That is, it is based on the comparison between the expected manoeuvre at time $t$ and the intended manoeuvre at time $t-1$. Table I shows the conditional probabilities. The evolution model assumes that drivers mostly respect traffic rules. In that way, the variable $P_{\text {comply }}$ is defined to model how much drivers comply with rules. A low value set for $P_{\text {comply }}$ means that most of the time drivers do not respect rules, while a high value means that drivers usually respect rules. If the intended manoeuvre at time $t-1$ and the expected manoeuvre at time $t$ do not match, a uniform prior is assumed.

3) Expected Driver Reaction $E R_{t}^{n}$ : The evolution model of $E R_{t}$, the expectation that the driver is reacting at time $t$, depends on how early the driver usually reacts to a stop intersection. The moment at which a driver react depends on $\gamma$, the average acceleration that he will have to undergo to stop. The later the driver reacts, the higher the acceleration
TABLE I: Conditional probabilities describing the intended manoeuvre.

\begin{tabular}{|c|c|c|c|}
\hline$I_{t-1}$ & $E_{t}$ & $P\left(\left[I_{t}=\right.\right.$ go $\left.] \mid I_{t-1}, E_{t}\right)$ & $P\left(\left[I_{t}=\right.\right.$ stop $\left.] \mid I_{t-1}, E_{t}\right)$ \\
\hline \hline go & go & $P_{\text {comply }}$ & $1-P_{\text {comply }}$ \\
\hline go & stop & 0.5 & 0.5 \\
\hline stop & go & 0.5 & 0.5 \\
\hline stop & stop & $1-P_{\text {comply }}$ & $P_{\text {comply }}$ \\
\hline
\end{tabular}
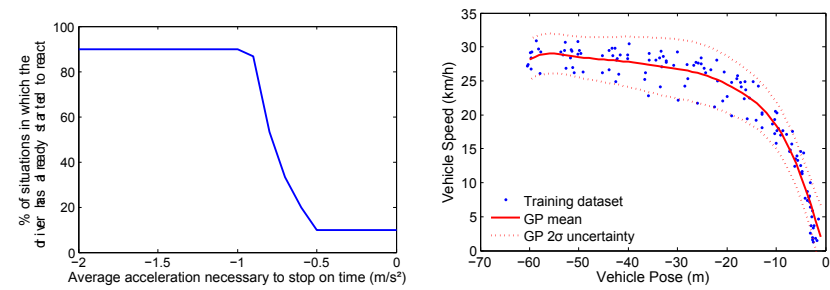

(a) Example of probability that the(b) Velocity profile learned using driver should be reacting Gaussian Processes

Fig. 2: Learnt driver patterns

that he has to undergo is. This acceleration depends on the driver driving style.

The approach that was chosen considers that drivers usually look to undergo accelerations that they are used to undergo, i.e. accelerations that they consider as comfortable. For each driver, it is necessary to learn when he usually starts to react to a stop intersection. For this purpose, the average accelerations that the driver usually do not have to undergo to stop are learnt. It enables to determine the relationship between average accelerations $\gamma$, and $\psi$ the percentage of situations in which the driver did not have to undergo average acceleration $\gamma$. This relationship is defined as the driver dependent function $f$, defined such as $\psi=f(\gamma)$. Figure $2 \mathrm{a}$ shows an example of function $f$ learnt for a particular driver.

4) Intended Driver Reaction $I R_{t}^{n}$ : The evolution model of $I R_{t}$, the driver's intention to react to the stop intersection at time $t$ is based on the comparison between the expected driver's reaction at time $t$ and the intended driver's reaction at time $t-1$. This model assumes that the driver mostly complies with the behaviour he is expected to have. This is represented through the parameter $P_{\text {comply }}$ that was presented before.

In that way, it is modelled that if intention at time $t-1$ and expectation at time $t$ are similar, the probability is high that intention at time $t$ is the same as the one at time $t-1$. Moreover, it is modelled that if the driver starts reacting at time $t-1$, the probability that he will keep on reacting at time $t$ is high. However, there is no prior assumption on intention at time $t$ when the driver was not reacting at time $t-1$ while he is expected to react at time $t$. Table II summarizes these conditional probabilities.

5) True Vehicle Pose $P_{t}^{n}$ : The evolution model of the true vehicle pose $P_{t}$ at time $t$ depends on the true vehicle pose $P_{t-1}$ and speed $S_{t-1}$ at time $t-1$. The conditional probability that describes $P_{t}$ can be simplified as follows: 
TABLE II: Conditional probabilities describing the intended driver reaction.

\begin{tabular}{|c|c|c|c|}
\hline$I R_{t-1}$ & $E R_{t}$ & $P\left(\left[I R_{t}=\right.\right.$ reaction $\left.] \mid I R_{t-1}, E R_{t}\right)$ & $P\left(\left[I R_{t}=\right.\right.$ noreaction $\left.] \mid I R_{t-1}, E R_{t}\right)$ \\
\hline \hline reaction & reaction & $P_{\text {comply }}$ & $1-P_{\text {comply }}$ \\
\hline reaction & noreaction & $P_{\text {comply }}$ & $1-P_{\text {comply }}$ \\
\hline noreaction & reaction & 0.5 & 0.5 \\
\hline noreaction & noreaction & $1-P_{\text {comply }}$ & $P_{\text {comply }}$ \\
\hline
\end{tabular}

$P\left(P_{t} \mid \phi_{t-1}, I_{t-1}, I_{t}\right)=P\left(P_{t} \mid P_{t-1}, S_{t-1}\right)=\mathcal{N}\left(\mu_{P}, \sigma_{P}^{\text {true }}\right)$

with:

- $\mu_{P}$ the mean of the pose. For the sake of simplicity, it is computed following a constant velocity model, using the value of $P_{t-1}$ and $S_{t-1}$, and the duration between timesteps $t-1$ and $t$.

- $\sigma_{P}^{\text {true }}$ the standard deviation of the pose in the motion model.

6) Observed Vehicle Pose Pobs $s_{t}^{n}$ : The evolution model of the observed vehicle pose Pobst at time $t$ is based on a classic sensor model as the measurements of the vehicle pose suffer from noise. The measurements follows a normal distribution centred on the true vehicle pose $P_{t}$. The conditional probability that describes $\mathrm{Pobs}_{t}$ can therefore be written as follows:

$$
P\left(\text { Pobs }_{t} \mid P_{t}\right)=\mathcal{N}\left(P_{t}, \sigma_{P}^{o b s}\right)
$$

7) True Vehicle Speed $S_{t}^{n}$ : The evolution model of the true vehicle speed $S_{t}$ at time $t$ depends on the true vehicle pose $P_{t-1}$ and speed $S_{t-1}$ at time $t-1$, and of the intended longitudinal manoeuvre at time t. $P_{t-1}$ and $S_{t-1}$ are used to predict the vehicle pose $P_{t}$ at time $t$, assuming constant speed between $t-1$ and $t . S_{t}$ is then based on the velocity profile corresponding to the intended longitudinal manoeuvre $I M_{t}$. These velocity profiles are assumed to follow normal distributions.

The likelihood of the true vehicle speed is defined as following a normal distribution such as:

$$
P\left(S_{t} \mid \phi_{t-1}, I_{t-1}, I_{t}\right)= \begin{cases}\mathcal{N}\left(\mu_{S}^{g o}, \sigma_{S}^{\text {go }}\right) & \text { if } I_{t}=g o \\ \mathcal{N}\left(\mu_{S}^{\text {stop }}, \sigma_{S}^{\text {stop }}\right) & \text { if } I_{t}=\text { stop }\end{cases}
$$

The assumption that all drivers are different is taken. For this purpose, the velocity profile representing the manner how the driver usually decelerates when he approaches to a stop intersection has to be customized. This was done using Gaussian Processes as it is described in [3]. Figure $2 b$ shows the example of a customized velocity profile for $I_{t}=$ stop . For $I_{t}=g o$, it is assumed that the vehicle speed is constant between $t-1$ and $t$, therefore $\mu_{S}^{g o}(t)=S_{t-1}$.

8) Observed Vehicle Speed Sobs $s_{t}^{n}$ : The measurements of the vehicle speed are collected from the vehicle CAN-bus, and do not suffer from uncertainties. They are modeled as follows:
TABLE III: Conditional probabilities describing the true state of the brake pedal.

\begin{tabular}{|c|c|c|}
\hline$I R_{t}$ & $P\left(\left[B_{t}=\right.\right.$ on $]\left[I R_{t}\right)$ & $P\left(\left[B_{t}=\right.\right.$ off $]\left[I R_{t}\right)$ \\
\hline \hline reaction & $P_{\text {comply }}$ & $1-P_{\text {comply }}$ \\
\hline noreaction & 0.5 & 0.5 \\
\hline
\end{tabular}

TABLE IV: Conditional probabilities describing the true state of the gas pedal.

\begin{tabular}{|c|c|c|}
\hline$I R_{t}$ & $P\left(\left[G_{t}=\right.\right.$ on $\left.] \mid I R_{t}\right)$ & $P\left(\left[G_{t}=\right.\right.$ off $\left.] \mid I R_{t}\right)$ \\
\hline \hline reaction & 0.5 & 0.5 \\
\hline noreaction & $P_{\text {comply }}$ & $1-P_{\text {comply }}$ \\
\hline
\end{tabular}

$$
P\left(\text { Sobs }_{t} \mid S_{t}\right)=\delta\left(S_{t}-\text { Sobs }_{t}\right)
$$

9) True State of Brake Pedal $B_{t}^{n}$ : The evolution model of $B_{t}$, the state of the brake pedal at time $t$ is based on $I R_{t}$, the intention to react at time $t$. The model assumes that pushing the brake pedal is a sign of reaction. It means that if the driver intends to react, the probability that he pushes the brake pedal is high. The parameter $P_{\text {comply }}$ which was presented before, is used to model this probability. However, if the driver does not intend to react, no prior assumption is done about the state of the brake pedal. Table III summarizes these conditional probabilities.

10) Observed State of Brake Pedal Bobs $s_{t}^{n}$ : The measurements of the state of the brake pedal are collected from the vehicle CAN-bus, and do not suffer from uncertainties. They are modeled as follows:

$$
P\left(\text { Bobs }_{t} \mid B_{t}\right)=\delta\left(B_{t}-\text { Bobs }_{t}\right)
$$

11) True State of Gas Pedal $G_{t}^{n}$ : The evolution model of $G_{t}$, the state of the gas pedal at time $t$ is based on $I R_{t}$, the intention to react at time $t$. The model assumes that pushing the gas pedal is a sign of no reaction. It means that is the driver pushes the gas pedal, the probability that he is not reacting is high. The parameter $P_{\text {comply }}$ which was presented before is used to model this probability. However, if the driver intends to react, no prior assumption is done on the state of the gas pedal. Table IV summarizes these conditional probabilities.

12) Observed State of Gas Pedal Gobs ${ }_{t}^{n}$ : The measurements of the state of the gas pedal are collected from the vehicle CAN-bus, and do not suffer from uncertainties. They are modeled as follows:

$$
P\left(G o b s_{t} \mid G_{t}\right)=\delta\left(G_{t}-\text { Gobs }_{t}\right)
$$




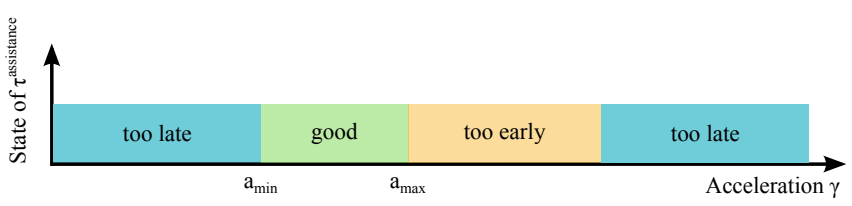

Fig. 3: Determination of the state of $\tau_{t}^{\text {assistance }}$ given $\gamma$.

TABLE V: Time and physical constraints

\begin{tabular}{|c|c|c|c|}
\cline { 2 - 4 } \multicolumn{1}{c|}{} & Reaction Time $\boldsymbol{R T}$ & $\begin{array}{c}\text { Minimum tolerated } \\
\text { acceleration } a_{\min }\end{array}$ & $\begin{array}{c}\text { Maximum tolerated } \\
\text { acceleration } a_{\max }\end{array}$ \\
\hline Automatic Braking & $R T_{\text {machine }}$ & $a_{\text {min }}^{\text {hard }}$ & $a_{\text {max }}^{\text {hard }}$ \\
\hline Warning & $R T_{\text {machine }}+R T_{\text {driver }}$ & $a_{\text {min }}^{\text {hard }}$ & $a_{\text {max }}^{\text {hard }}$ \\
\hline Advice & $R T_{\text {machine }}+R T_{\text {driver }}$ & $a_{\text {min }}^{\text {smooth }}$ & $a_{\text {max }}^{\text {smooth }}$ \\
\hline
\end{tabular}

13) Assistance $A_{t}^{n}=\left(A c t_{t}^{n}, \operatorname{War}_{t}^{n}, A d v_{t}^{n}\right)$ : Assistance is necessary if a risk has been detected. However, the choice of the type of assistance that has to be provided depends on the constraints imposed by the vehicle state. Actually, providing an advice does not make sense if after reaction, the driver has to undergo a too hard deceleration. Moreover, performing automatic braking is not pertinent if the deceleration that the vehicle has to undergo is too smooth. Conditions have therefore to be specified to estimate if a given type of assistance would be pertinent at time $t$, with respect to the vehicle state.

For this purpose, indicator $\tau_{t}^{\text {assistance }} \in$ $\{$ good,toolate, too early\}, with assistance $\in$ \{advice,warning,actuation\} is defined in order to evaluate the pertinence of each type of assistance in case of risky situations. It indicates whether it would be time, too late or too early to provide assistance. The three indicators $\tau_{t}^{\text {advice }}, \tau_{t}^{\text {warning }}$ and $\tau_{t}^{\text {actuation }}$ are defined, respectively for advice assistance, warning assistance and AEB assistance.

The state of $\tau_{t}^{\text {assistance }}$ depends on:

- The value of the average acceleration $\gamma$ that the vehicle would have to undergo to stop on time if assistance was provided. This acceleration is computed by Equation 8 , considering the vehicle true speed $S_{t}$, the vehicle true pose $P_{t}$ and reaction time $R T$. The reaction time includes the driver's reaction time and the vehicle response.

- An acceleration interval defined by the variables $a_{m i n}$ and $a_{\max }$. Figure 3 presents how $\gamma$ is compared to this interval to get the state of $\tau_{t}^{\text {assistance. Table } \mathrm{V}}$ presents the time and physical constraints imposed by each type of assistance. Note that warning and AEB assistance allow for harder acceleration (low values of $\gamma$, with $\gamma<0$ ) than advice assistance.

$$
\gamma=-\frac{S_{t}^{2}}{2\left(P_{t}-S_{t} \cdot R T\right)}
$$

The pertinence at time $t$ of each type of assistance for the driver of vehicle $n$ is defined as follows. a) Advice $A d v_{t}^{n}$ : A behaviour is considered as suspicious when the driver does not show any sign leading to think that he is aware that he has to stop at the intersection. This is done by comparing the intended driver reaction $I R_{t}$ and the expected driver reaction $E R_{t}$. In that way, the driver's behaviour is considered as suspicious at time $t$ as soon as $I R_{t}=$ noreaction and $E R_{t}=$ reaction. Moreover, the state of indicator $\tau_{t}^{\text {advice }}$ has to be good.

The probability that it would be pertinent to provide the driver with an advice is given by:

$$
\begin{aligned}
& P\left(\left[\text { Adv } v_{t}=\text { pertinent }\right] \mid I R_{t}, E R_{t}, \tau_{t}^{\text {advice }}\right) \\
& =\left\{\begin{array}{c}
1 \text { if }\left\{\begin{array}{l}
{\left[\text { I } R_{t}=\text { no reaction }\right],\left[E R_{t}=\text { reaction }\right], \ldots} \\
{\left[\tau_{t}^{\text {advice }}=\text { good }\right]}
\end{array}\right. \\
0 \text { otherwise }
\end{array}\right.
\end{aligned}
$$

b) Warning $\operatorname{War}_{t}^{n}$ : It is considered that warning the driver is pertinent as soon as these two conditions are satisfied at the same time:

- The comparison between the driver's intended reaction $I R_{t}$ with the expected reaction $E R_{t}$ shows that the driver's behaviour is suspicious, i.e. the driver does not react while he is expected to react. Moreover, the state of indicator $\tau_{t}^{\text {warning }}$ has to be good.

- Risk has been detected by comparing the intended vehicle manoeuvre $I M_{t}$ with the expected one, $E M_{t}$, i.e. by inferring that the driver does not have intention to stop while he is expected to stop.

The probability that it would be pertinent to provide the driver with warning assistance is given by:

$$
\begin{aligned}
& P\left(\left[\text { War }_{t}=\text { pertinent }\right] \mid I R_{t}, E R_{t}, I M_{t}, E M_{t}, \tau_{t}^{\text {warning }}\right) \\
& =\left\{\begin{array}{c}
1 \text { if }\left\{\begin{array}{l}
{\left[I R_{t}=\text { no reaction }\right],\left[E R_{t}=\text { reaction }\right], \ldots} \\
{\left[I M_{t}=\text { go }\right],\left[E M_{t}=\text { stop }\right], \ldots} \\
{\left[\tau_{t}^{\text {warning }}=\text { good }\right]}
\end{array}\right. \\
0 \text { otherwise }
\end{array}\right.
\end{aligned}
$$

c) Actuation $A c t_{t}^{n}$ : Risk is estimated by comparing the intended vehicle manoeuvre $I M_{t}$ with the expected one, $E M_{t}$. It is considered that if the last solution to avoid an accident is last minute automatic emergency braking, then any sign of driver reaction does not cast doubt on the pertinence of this assistance. Thus, the variables $I R_{t}$ and $E R_{t}$ describing respectively the intended and expected driver reactions are not considered. Moreover, the state of indicator $\tau_{t}^{\text {actuation }}$ has to be good.

The probability that it would be pertinent to provide the driver with an AEB is given by:

$$
\begin{aligned}
& P\left(\left[\text { Act }_{t}=\text { pertinent }\right] \mid I M_{t}, E M_{t}, \tau_{t}^{\text {actuation }}\right) \\
& =\left\{\begin{array}{c}
1 \text { if }\left\{\begin{array}{l}
{\left[I M_{t}=\text { go }\right],\left[E M_{t}=\text { stop }\right], \ldots} \\
{\left[\tau_{t}^{\text {actuation }}=\text { good }\right]}
\end{array}\right. \\
0 \text { otherwise }
\end{array}\right.
\end{aligned}
$$


14) Joint Probability: The joint probability of the BN can be simplified as follows:

$$
\begin{aligned}
& P\left(A_{0: T}, E_{0: T}, I_{0: T}, \phi_{0: T}, Z_{0: T}\right) \\
& =P\left(A_{0}, E_{0}, I_{0}, \phi_{0}, Z_{0}\right) \cdot \prod_{t=1}^{T} P\left(E M_{t} \mid I_{t-1}, \phi_{t-1}\right) \\
& \cdot P\left(E R_{t} \mid P_{t-1}, S_{t-1}\right) \cdot P\left(I M_{t} \mid \phi_{t-1}, I_{t-1}, E_{t}\right) \\
& \cdot P\left(I R_{t} \mid I R_{t-1}, E R_{t}\right) \cdot P\left(S_{t} \mid P_{t-1}, S_{t-1}, I_{t}\right) \\
& \cdot P\left(P_{t} \mid P_{t-1}, S_{t-1}\right) \cdot P\left(B_{t} \mid I R_{t}\right) \cdot P\left(G_{t} \mid I R_{t}\right) \\
& \cdot P\left(\operatorname{Sobs}_{t} \mid S_{t}\right) \cdot P\left(P o b s_{t} \mid P_{t}\right) \cdot P\left(\text { Bobs }_{t} \mid B_{t}\right) \cdot P\left(G o b s_{t} \mid G_{t}\right) \\
& \cdot P\left(A c t_{t} \mid E M_{t}, I M_{t}, S_{t}, P_{t}\right) \\
& \cdot P\left(A d v_{t} \mid E R_{t}, I R_{t}, E M_{t}, I M_{t}, S_{t}, P_{t}\right) \\
& \cdot P\left(A d v_{t} \mid E R_{t}, I R_{t}, S_{t}, P_{t}\right)
\end{aligned}
$$

\section{Results AND Discussion}

\section{A. Experimental Setup}

1) Facilities: A passenger vehicle driven on closed road was used for the experimental part. The vehicle speed, and the state of the brake and gas pedals were extracted from the vehicle CAN bus. The vehicle position was estimated from an automotive type GPS receiver running at $5 \mathrm{~Hz}$ (Ublox). A navigation system which takes benefits from Open Street Map digital maps was used. It allows to perform map matching according to the vehicle position, and to extract the distance remaining before the vehicle reaches the stop intersection.

2) Data Acquisition and Inferences: A dataset containing 260 runs, recorded with 4 different drivers, with different styles was created. In this dataset, 130 runs were labeled safe as the driver intends to stop, and 130 runs were labeled risk as the driver does not intend to stop. For each recorded run, the following probabilities were computed through the Bayesian network:

- $P\left(\left[A c t_{t}=\right.\right.$ pertinent $\left.] \mid S m_{0: t}, P m_{0: t}, B m_{0: t}, G m_{0: t}\right)$, the probability that performing automatic actuation at time $t$ is pertinent.

- $P\left(\left[\right.\right.$ War $_{t}=$ pertinent $\left.] \mid S m_{0: t}, P m_{0: t}, B m_{0: t}, G m_{0: t}\right)$, the probability that providing a warning to the driver at time $t$ is pertinent.

- $P\left(\left[A d v_{t}=\right.\right.$ pertinent $\left.] \mid S m_{0: t}, P m_{0: t}, B m_{0: t}, G m_{0: t}\right)$, the probability that providing an advice to the driver at time $t$ is pertinent.

All inferences were performed using the following parameters: $P_{\text {comply }}=0.9, \sigma_{P}^{\text {obs }}=3 \mathrm{~m}, \sigma_{P}^{\text {true }}=1 \mathrm{~m}$, $R T_{\text {machine }}=0.4 \mathrm{~s}, R T_{\text {driver }}=1.5 \mathrm{~s}, a_{\text {min }}^{\text {hard }}=-8 \mathrm{~m} / \mathrm{s}^{2}$, $a_{\text {max }}^{\text {hard }}=-5 \mathrm{~m} / \mathrm{s}^{2}$. The values of $a_{\text {min }}^{\text {smoth }}$ and $a_{\text {max }}^{\text {smooth }}$ depend on the drivers' style. Average values are $a_{\text {min }}^{\text {smooth }}=$ $-3 \mathrm{~m} / \mathrm{s}^{2}$ and $a_{\max }^{\text {smooth }}=-1.5 \mathrm{~m} / \mathrm{s}^{2}$. The inferences were performed through a particle filter running with $N=400$ particles.

\section{B. Qualitative Results}

Figure 4 present the inferences obtained in the case of a safe situation, and in the case of a risk situation.

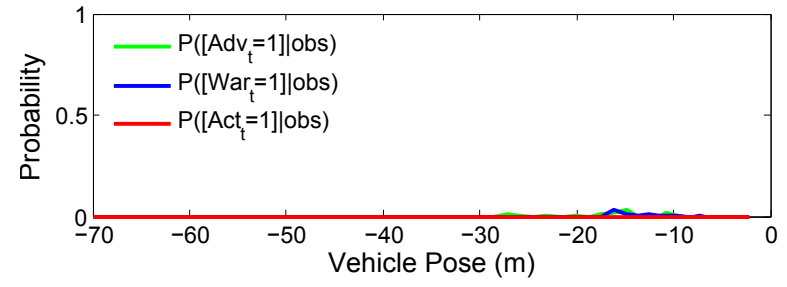

(a) Safe situation

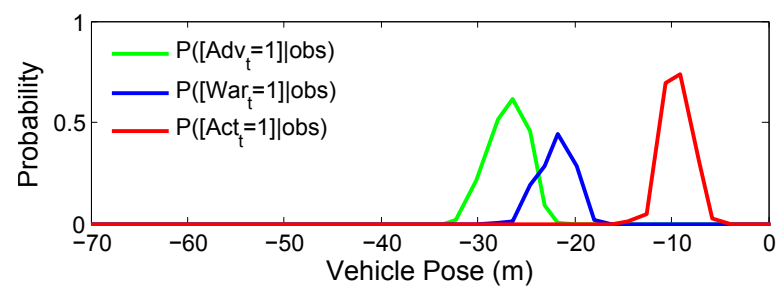

(b) Risk situation

Fig. 4: Qualitative results.

1) Safe Situation: Figure 4a shows, in the case of a safe situation, the probabilities that it would be pertinent to provide the driver with each type of assistance, namely automatic braking, warning and advice. It is noticeable that these three probabilities stay low all over the run, meaning that providing the driver with any assistance is not pertinent. This is explained by the fact that the driver properly reacts and decelerates as he is expected to do.

2) Risk Situation: Figure $4 \mathrm{~b}$ shows, in the case of a safe situation, the probabilities that it would be pertinent to provide the driver with each type of assistance, namely automatic braking, warning and advice. It is noticeable that, as the driver does not react and decelerate as expected, these three probabilities make peaks at different distances to intersection:

- $P\left(\left[\right.\right.$ Act $_{t}=$ pertinent $\left.] \mid S m_{0: t}, P m_{0: t}, B m_{0: t}, G m_{0: t}\right)$ is significant from $P_{t} \simeq 15 \mathrm{~m}$ to $P_{t} \simeq 5 \mathrm{~m}$, meaning that is would be pertinent to provide the driver with automatic braking as long as the vehicle is located from 15 to $5 \mathrm{~m}$ to the intersection.

- $P\left(\left[\right.\right.$ War $_{t}=$ pertinent $\left.] \mid S m_{0: t}, P m_{0: t}, B m_{0: t}, G m_{0: t}\right)$ is significant from $P_{t} \simeq 25 \mathrm{~m}$ to $P_{t} \simeq 15 \mathrm{~m}$, meaning that is would be pertinent to warn the driver as long as the vehicle is located from 25 to $15 \mathrm{~m}$ to the intersection.

- $P\left(\left[A d v_{t}=\right.\right.$ pertinent $\left.] \mid S m_{0: t}, P m_{0: t}, B m_{0: t}, G m_{0: t}\right)$ is significant from $P_{t} \simeq 30 \mathrm{~m}$ to $P_{t} \simeq 20 \mathrm{~m}$, meaning that is would be pertinent to provide the driver with an advice as long as the vehicle is located from 30 to $20 \mathrm{~m}$ to the intersection.

\section{Quantitative Results}

The inferences aim to be used to estimate whether or not assistance is necessary. To trigger assistance, the probability that assistance is pertinent has to be greater than a threshold $\lambda$. The dataset was used to compute Recall and Precision, 
TABLE VI: Performances of the BN for triggering assistance

\begin{tabular}{|c|c|c|c|}
\cline { 2 - 4 } \multicolumn{1}{c|}{} & TP (\%) & FP (\%) & $\left(\mu_{\gamma}, \sigma_{\gamma}\right)$ \\
\hline Auto Braking & 100 & 0 & $(-4.72,0.43)$ \\
\hline Warning & 84 & 0 & $(-5.09,1.01)$ \\
\hline Advice & 82 & 4 & $(-2.64,0.45)$ \\
\hline
\end{tabular}

in order to identify the optimized threshold for each type of assistance (which minimize the rate of false positive (FP) and maximize the rate of true positive (TP)). In that way, $\lambda_{\text {actuation }}=0.2, \lambda_{\text {warning }}=0.15$ and $\lambda_{\text {advice }}=0.3$ were found as optimized values, respectively for automatic actuation, warning and advice assistance.

Table VI presents the performances which were reached for the three types of assistance: rate of true positive (TP), rate of false positive (FP) and the acceleration that the vehicle would undergo to stop on time, taking into consideration the reaction time of the driver and of the machine.

- Automatic braking can be triggered $100 \%$ of the time it is necessary, without false alarm. The average acceleration that the vehicle would undergo is $-4.72 \mathrm{~m} / \mathrm{s}^{2}$.

- Warning assistance can be triggered $84 \%$ of the time it is necessary, without false alarm. The average acceleration that the vehicle would undergo is $-5.09 \mathrm{~m} / \mathrm{s}^{2}$. Note that acceleration is lower that the one obtained with automatic braking. This can be explained by $\lambda_{\text {actuation }}>\lambda_{\text {warning }}$, implying longer time to trigger Warning Assistance, and thus harder deceleration.

- Advice assistance can be triggered $82 \%$ of the time it is necessary, with $4 \%$ of false alarm. The average acceleration that the vehicle would undergo is $-2.64 \mathrm{~m} / \mathrm{s}^{2}$.

\section{Discussion}

The results obtained with the dataset show that the proposed $\mathrm{BN}$ allows to infer risk situations and to trigger the three types of assistance at the most suitable moment. In the case of automatic actuation (AEB) and warning assistance (curative assistance), it is shown that assistance can be provided without risk of inopportune assistance, and allowing the vehicle or the driver to brake with realistic emergency deceleration.

Moreover, it is shown that the $\mathrm{BN}$, by taking into consideration the vehicle state, the driver actuation and individualities, allows to detect that the situation is likely to become at risk, whilst it is not yet dangerous. Further, advice assistance (preventive) triggered by this BN inferences would allow the driver to have time to react, and to decelerate comfortably after being provided with assistance.

The results which were presented are still theoretical and need to be confirmed with field trials in order to collect the drivers feedback about the pertinence of the provided advices. For this purpose, a dedicated Human Machine Interface (HMI) needs to be developed to provide information to the driver.

\section{CONClusion}

This paper proposed a Bayesian Network which allows to infer risk situations, and to identify at any time which type of assistance is the most pertinent to be provided. The BN takes into consideration the context, the vehicle state and the driver actuation. Further, it considers that all drivers may react differently for a same situation, and thus takes benefits from customized drivers profiles to best adapt to the driver's style. The model was applied to the approach to stop intersections. Results, generated using data recorded in a passenger vehicle driven by several drivers, show that the $\mathrm{BN}$ allows to detect risk situations, and to trigger coherently automatic braking and warning assistance. Further, it infers risks early enough to trigger preventive assistance which could be provided in the form of advice.

Further work should consist in taking into consideration other vehicles approaching to the same intersection, and which could have conflicting intention with the subject vehicle. This would imply to change the parametric form of the manoeuvres expectation, to make it depend on the context and on the state of the other vehicle, as it was done in [7].

\section{REFERENCES}

[1] Samer Ammoun and Fawzi Nashashibi. Real time trajectory prediction for collision risk estimation between vehicles. In Intelligent Computer Communication and Processing, 2009. ICCP 2009. IEEE 5th International Conference on, pages 417-422. IEEE, 2009.

[2] G.S. Aoude, V.R. Desaraju, L.H. Stephens, and J.P. How. Driver behavior classification at intersections and validation on large naturalistic data set. in Proceedings of the IEEE Intelligent Transportation Systems Conference, 13(2):724-736, june 2012.

[3] A. Armand, D. Filliat, and J. Ibañez-Guzmán. Modelling stop intersection approaches using gaussian processes. In Proceedings of the 16th International IEEE Conference on Intelligent Transportation Systems-ITSC, 2013.

[4] H. Berndt, J. Emmert, and K. Dietmayer. Continuous driver intention recognition with hidden markov models. in Proceedings of the IEEE Intelligent Transportation Systems Conf., pages 1189 -1194, oct. 2008.

[5] Sarah Bonnin, Thomas H Weisswange, Franz Kummert, and Jens Schmuedderich. General behavior prediction by a combination of scenario-specific models. IEEE transactions on intelligent transportation systems, 15(4):1478-1488, 2014.

[6] Tay Christopher. Analysis of dynamic scenes: Application to driving assistance. PhD thesis, Institut National Polytechnique de GrenobleINPG, 2009.

[7] Stéphanie Lefevre. Risk estimation at road intersections for connected vehicle safety applications. PhD thesis, Inria Grenoble Rhône-Alpes, 2012.

[8] Andrew Naftel and Shehzad Khalid. Classifying spatiotemporal object trajectories using unsupervised learning in the coefficient feature space. Multimedia Systems, 12(3):227-238, 2006.

[9] European Road Safety Observatory. Traffic safety basic facts 2010 junctions. SafetyNet, Project co-financed by the European Commission, 2010.

[10] M.G. Ortiz, J. Fritsch, F. Kummert, and A. Gepperth. Behavior prediction at multiple time-scales in inner-city scenarios. in Proceedings of the IEEE Intelligent Vehicles Symposium, pages 1068 -1073, june 2011.

[11] Robin Schubert, Eric Richter, and Gerd Wanielik. Comparison and evaluation of advanced motion models for vehicle tracking. In Information Fusion, 2008 11th International Conference on, pages 1-6. IEEE, 2008.

[12] TRACE. Analyzing human factors in road accidents. Technical report, 2008. 